\title{
Rapid and mask-less laser-processing technique for the fabrication of microstructures in polydimethylsiloxane
}

\author{
C. L. Sones, ${ }^{*}$ I. N. Katis, B. Mills, M. Feinäugle, A. Mosayyebi,
}

J. Butement, and R. W. Eason

Optoelectronics Research Centre, University of Southampton, Southampton, SO17 1BJ , U.K.

\begin{abstract}
We report a rapid laser-based method for structuring polydimethylsiloxane (PDMS) on the micron-scale. This mask-less method uses a digital multi-mirror device as a spatial light modulator to produce a given spatial intensity pattern to create arbitrarily shaped structures via either ablation or multi-photon photo-polymerisation in a master substrate, which is subsequently used to cast the complementary patterns in PDMS. This patterned PDMS mould was then used for micro-contact printing of ink and biological molecules.
\end{abstract}

\section{Keywords}

Ablation

Photo-polymerisation

Digital multi-mirror

Laser material processing

Microstructure fabrication

Micro-contact printing

${ }^{*}$ Corresponding author- email: cls@orc.soton.ac.uk

Tel: $(+44) 02380593141$

Fax: (+44) 02380593142 


\subsection{Introduction}

Polydimethylsiloxane (PDMS) finds widespread use as the substrate that forms the platform for the fabrication of microfluidics-based lab-on-chip devices [1,2]. It is also a material of choice in micro-contact printing for the creation of a mould that is then repeatedly used for stamping an array of specifically desired biological molecules [3, 4]. The popularity of PDMS in such biological application stems from some of its distinctive characteristics which include biocompatibility, elasticity (which allows it to be flexibly moulded into the desired shape and be released from a rigid and complex 3D master mould), optical transparency down to $\sim 230$ $\mathrm{nm}$, chemical and thermal stability, isotropic nature, homogeneity, durability and low-cost [13]. These characteristics lead to its selection as a material of choice for microfluidics-based lab-on-chip devices and applications in micro-contact printing. However, its ability to be moulded or structured is the key advantage, as this allows the formation of embedded structures, such as fluidic channels, and surface relief structures, such as upstanding blocks or pillars.

A range of methods such as wet-chemical etching, reactive-ion etching [5, 6], light induced patterning [7], decal transfer microlithography [8], the bond-detach method [9], and printand-peel [10] approaches (such as photo-copying [6] and solid-object printing [11, 12]) have been utilised for creating patterns in PDMS through the use of a master. However for the former case, one of the most commonly used approaches for the structuring of PDMS for such applications is through the creation of a pattern in either a photoresist (SU8), or in a film of $\mathrm{SiO}_{2}$ or $\mathrm{Si}_{3} \mathrm{~N}_{4}$ or metal on a $\mathrm{Si}$ or $\mathrm{SiO}_{2}$ substrate to produce a master mould that is used to cast the PDMS [1, 3, 13]. This more often than not involves the use of a clean-room based UVlithographic procedure that utilizes custom-designed masks for the creation of a mastermould. The structures that are formed in the master are then duplicated by casting PDMS on it to produce a secondary-mould of PDMS - which is then used for end applications in microcontact printing or, by itself as a fluidic substrate.

Even though this lithographic procedure can routinely produce high-resolution micron-scale structures, the procedure, by its very nature, is both expensive and time-consuming. Instead, in this paper, we demonstrate, for the production of the master and hence the secondary PDMS-mould, a rapid and flexible alternative to the expensive UV-lithographic procedure. More recently, techniques describing the structuring of PDMS directly, using both ultra-short pulsed lasers for laser-induced thermal curing [14] and through the addition of photoinitiators in PDMS via avalanche ionisation [15, 16], have also been reported. The method here is a mask-less laser-based direct-write procedure that does not rely on cleanroom access. As in soft-lithography, the creation of the PDMS-mould via the mask-less approach shown here, occurs in two steps, and allows the creation of high-quality micron to millimetre-scale features in PDMS. The process is generic, and not limited to PDMS, but can also be used to create structures in a wide range of other materials, with the added advantage of being able to 
fabricate complex and differently-shaped structures adjacent to one another, in a single laser patterning step. Furthermore, through a simple step-and-repeat procedure it is then possible to stitch together a 2D array of complex patterns over a larger area of several $\mathrm{cm}^{2}$.

This laser-based direct-write method uses a digital multi-mirror device (DMD) as an intensity spatial light modulator in order to produce a user-defined laser light pattern for the creation of the complex structures. The use of a spatial light modulator enables the laser-processing to be parallelised, as an entire pattern can be fabricated at once, hence enabling a speed advantage over point-by-point serial-process writing techniques. In this work, the mastermould with user-defined pattern was created by either laser-ablation or multiphoton polymerisation [17-20], which are commonly employed laser-based techniques extensively used in materials patterning. Patterns that are complementary to the two-dimensional surface relief patterns created in the master-mould were then produced in a PDMS mould. To demonstrate the applicability of this laser-based procedure, this paper also presents the use of a laser-patterned PDMS mould for micro-contact printing of ink and fluorescently-tagged biomolecules.

\subsection{Experimental set-up and methods}

Figure 1 shows a schematic of the laser-based patterning set-up used for this work, and more details about it can be found in [21]. The laser used was a Ti:sapphire ultrafast amplifier (Coherent Legend-F, seeded by a Coherent Mira-900 oscillator), with a central wavelength of $800 \mathrm{~nm}$, pulse length of $150 \mathrm{fs}$, repetition rate of $1 \mathrm{kHz}$, and a pulse energy of $2 \mathrm{~mJ}$.Patterning was effected by reflecting the laser light from a digital multi-mirror device, which is a pixelated programmable mirror array from Texas Instruments (DLP3000) [22] that has an array of $608 \times 684$ mirrors, each of width of $\sim 7.6 \mu \mathrm{m}$ and horizontal and vertical centre-tocentre distances of $10.8 \mu \mathrm{m}$. Each mirror in the array was individually programmed to either turn 'on' or 'off' (by loading a static black and white bitmap onto the DMD), corresponding to a change in the angular deviation of the mirrors on their supporting-hinge by an angle of either $+12^{\circ}$ or $-12^{\circ}$ with respect to the surface of the device. This pattern was then used for subsequent laser machining of the sample, with a typical fluence of $\sim 1.7 \mathrm{~J} / \mathrm{cm}^{2}$ (corresponding to a peak power of $11 \mathrm{TW} / \mathrm{cm}^{2}$ ). The pulse energy before the 50x objective, as shown in Fig. 1, was $\sim 17 \mu \mathrm{J}$. 


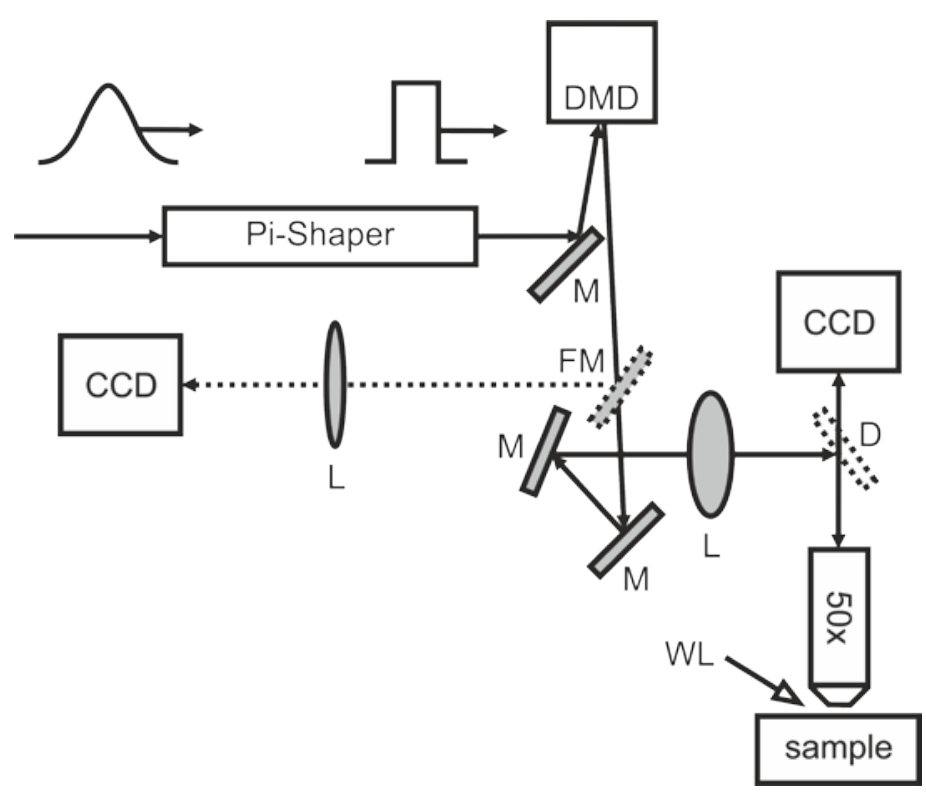

Figure 1: Schematic of the DMD-based laser patterning set-up.

In order to ensure illumination of the DMD by a uniform light intensity pattern, the Gaussian spatial beam profile of the laser was homogenised and thus transformed into a top-hat intensity profile using a refractive element ( $\pi$-shaper, model 6_6, from www.pishaper.com). As shown in Fig.1, using appropriate relay optics (mirrors ' $\mathrm{M}$ ', flip mirrors ' $F M$ ', lenses 'L', dielectric mirror ' $\mathrm{D}$ ') and imaging optics (a 50x objective, NA=0.55, SLMP, Nikon) the intensity pattern immediately after the DMD was de-magnified onto the sample. A co-axial imaging setup that used a white light (WL) source and a CCD camera allowed real-time observation of the DMD-assisted laser-machining of the work-piece. The sample was mounted on a computer-controlled three-axis translation stage to allow precise positioning of the sample within the image-plane, and for stitching together an array of individually created patterns. Using this DMD-based laser-machining set-up it was possible to pattern an area of $\sim 30 \mu \mathrm{m} \times 30 \mu \mathrm{m}$ via a single laser pulse. This area was determined by the demagnification optics positioned between the DMD and the target sample, which were chosen to find a balance between a larger area (enabling larger areas to be laser-processed at once) and a smaller area (providing a higher intensity). However, when combined with step-and-repeat techniques, at laser repetition rates of $1 \mathrm{kHz}$ and single pulse energies of $\sim 1 \mathrm{~mJ}$, it was possible to achieve a final patterned area of around $1 \mathrm{~cm}^{2}$ (with micron-scale feature sizes) within reasonable (less than 1 hour) time scales. Whilst using a step-and-repeat process can introduce stitching errors, we found that the use of high-precision stages, with sub-micron accuracy, and the use of automated backlash compensation appeared to make this error negligible.

In standard UV-photolithography, in addition to the time required for the fabrication of a custom-designed mask, the subsequent steps of spinning, patterning, developing, deposition or evaporation and wet-etching can be time-consuming, and will then produce only dedicated pre-designed patterns. As a direct comparison with regards to cost and time, the approach 
presented here is a mask-less procedure, with complete freedom over feature design, and hence the cost, availability and fabrication of designer or 'one-off' structures compares very favourably.

In order to create a master-mould with the desired set of patterns, experiments using two distinct approaches (Fig.2) namely laser-based ablation and laser-based multiphoton polymerisation have been performed.

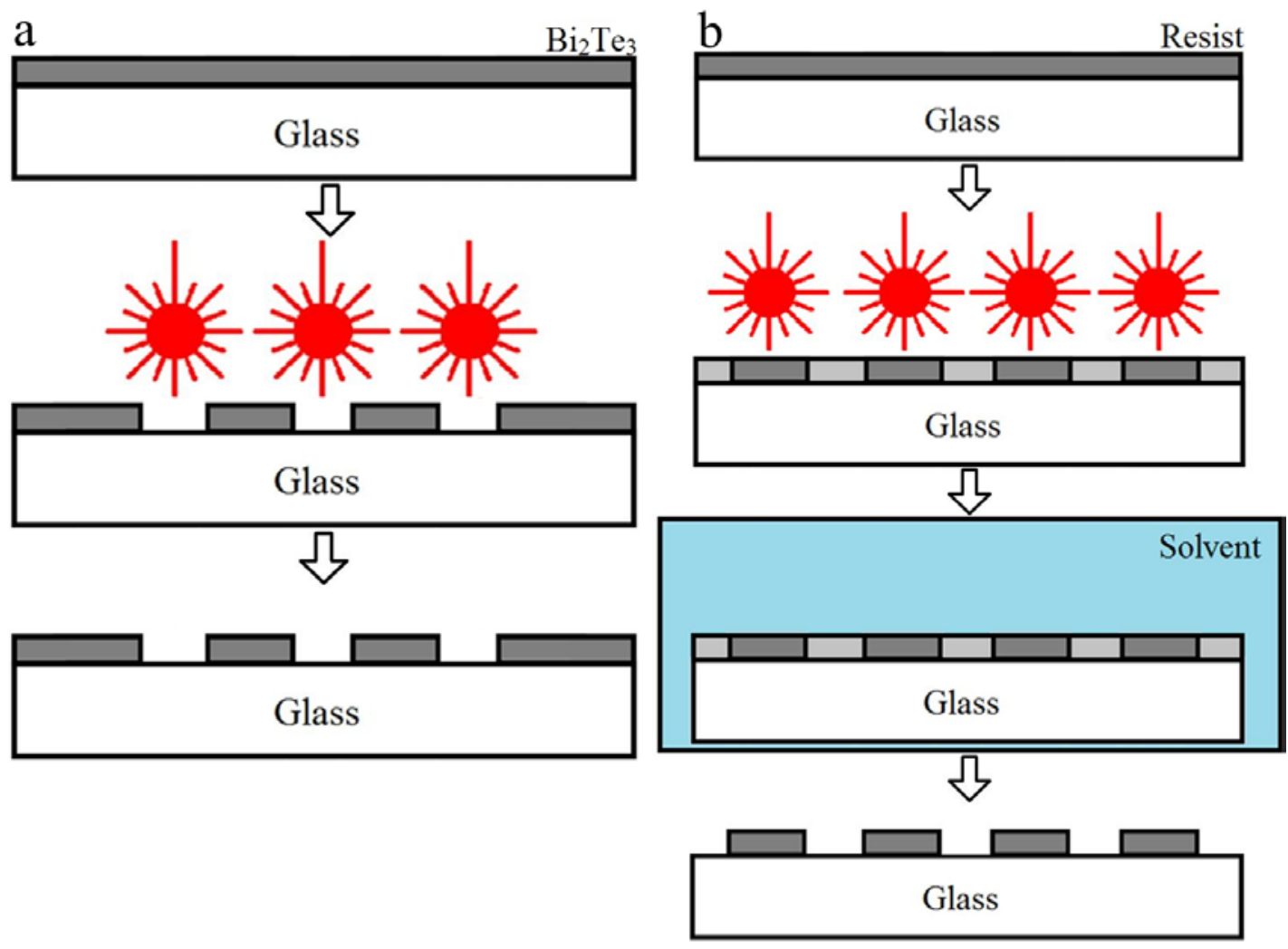

Figure 2: Schematic of the two DMD-based routes used to pattern a master via (a) ablation, and (b) multiphotonpolymerisation of the DMD-based laser patterning set-up.

\subsection{Results and discussion}

\subsection{Laser-based ablative patterning}

For the first case we chose to fabricate the master via ablation of $\mathrm{a} \sim 1 \mu \mathrm{m}$ thick bismuth telluride $\left(\mathrm{Bi}_{2} \mathrm{Te}_{3}\right)$ film that had been sputter-coated onto a glass substrate, although any material that can be ablated could have been used. The first set of experiments included ablation of a two-dimensional array of $10 \times 10$ square trenches, with each square having a dimension of $30 \mu \mathrm{m} \times 30 \mu \mathrm{m}$ and a centre-to-centre separation of $\sim 50 \mu \mathrm{m}$. Each square 
trench was ablated in the $\mathrm{Bi}_{2} \mathrm{Te}_{3}$ film using 10 sequential laser pulses with a fluence of 1.7 $\mathrm{J} / \mathrm{cm}^{2}$ (corresponding to a peak power of $11 \mathrm{TW} / \mathrm{cm}^{2}$ ). We chose to create an array of simple square patterns as when casted with PDMS, the secondary-mould would have the complementary square pillars which are best suited for the subsequent contact printing experiments. Also, these initial set of experiments allowed us to identify the laser-conditions required for the creation of well-defined features. It was found that 10 pulses was the minimum number required to produce structures with shapes having good fidelity with the DMD-projected image pattern, good verticality and extended throughout the entire thickness of the $\mathrm{Bi}_{2} \mathrm{Te}_{3}$ film. The size of the features ablated were on the order of microns, however, using the same set-up we have been able to demonstrate features with sizes of $<400 \mathrm{~nm}$ [21].

To demonstrate the advantage offered by this direct-write procedure in the production of an array constituted of features with varied shapes and sizes, the second set of ablativepatterning experiments involved the ablation of different shapes (a square, a triangle and a circle) in a single step, via a different DMD pattern. The incident fluence was kept at $1.7 \mathrm{~J} / \mathrm{cm}^{2}$ (corresponding to a peak power of $11 \mathrm{TW} / \mathrm{cm}^{2}$ ) however 100 pulses were used to ablate the pattern-set. It should be noted that 10 pulses were sufficient to ablate the pattern-set and the additional pulses were employed as means to 'clean-up' any debris that might have remained on the underlying glass substrate. Multiple step-and-repeat ablations resulted in the formation of an array within ten minutes, with lateral dimensions of about few hundred microns. Fig.3a shows an image of DMD-ablated BiTe film acquired using an optical interference-based non-contact surface profiler (ZeScope from Zemetrics) [23]. The inset in Fig.3a shows the black/white bitmap pattern that was loaded onto the DMD. In this work, white regions of the bitmap correspond to the presence of laser light on the target sample, whilst black regions correspond to no laser light.
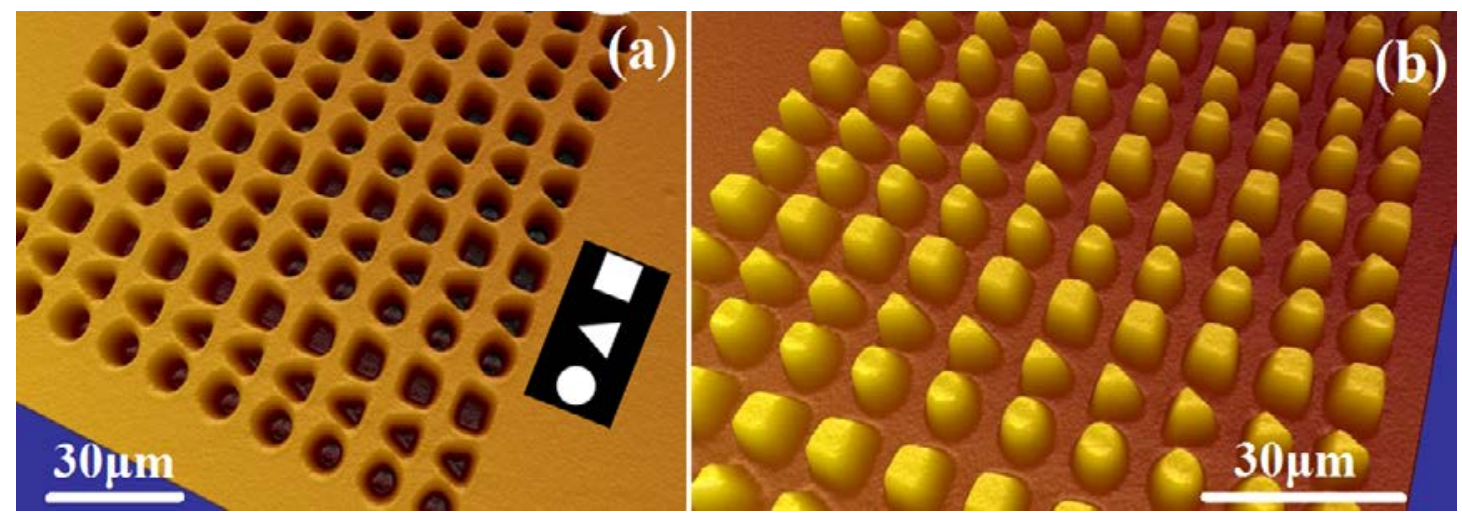

Figure 3: Images (a) shows a 2D array of $10 \times 30$ shapes formed in a $\sim 1 \mu m$ thick $\mathrm{Bi}_{2} \mathrm{Te}_{3}$ film via $\mathrm{DMD}$-ablation. The black/ white inset shows the bitmap loaded onto the $\mathrm{DMD}$ for ablation of the $\mathrm{Bi}_{2} \mathrm{Te}_{3}$ film; (b) shows a complementary 2D array of $10 \times 30$ shapes (circles, triangles and squares) formed in PDMS using the $\mathrm{Bi}_{2} \mathrm{Te}_{3}$ mould in figure 3(a). Images have been taken using an optical interference-based non-contact surface profiler. The depths of the ablated features, and hence the corresponding heights of the upstanding PDMS structures was measured to be $\sim 1 \mu \mathrm{m}$. They however appear to be $>1 \mu \mathrm{m}$ because the images have been adapted through the profiler software for better visualisation. 
These ablated patterns were then used to form a secondary-mould in PDMS. A 10:1 mixture of a PDMS base and a curing agent was mixed thoroughly, de-gassed to remove trapped air-bubbles, and poured onto the patterned $\mathrm{Bi}_{2} \mathrm{Te}_{3}$ film. The mixture was allowed to set at $90{ }^{\circ} \mathrm{C}$ for one hour. As shown in Fig.3b, this led to the creation of a complementary micron-scale two-dimensional pattern of upstanding structures in PDMS.

As the thickness of the $\mathrm{Bi}_{2} \mathrm{Te}_{3}$ film that was patterned through ablation via the $\mathrm{DMD}$ was of the order of $\sim 1 \mu \mathrm{m}$, the features formed in the PDMS mould also had heights of $\sim 1 \mu \mathrm{m}$, but features with greater heights can readily be formed from higher aspect ratio ablated mastermoulds.

\subsection{Laser-based photo-polymerisation}

In this section, we demonstrate an additional approach that relies on DMD-based multiphoton-polymerisation for the creation of the master-mould. This approach allowed us to produce patterns with feature depths (in the master) and heights (in the PDMS mould) of $10 \mu \mathrm{m}$. To validate this route we first spin coated a $\sim 10 \mu \mathrm{m}$ thick film of a photo-sensitive polymer (a silicon/zirconium hybrid negative resist) onto a glass substrate [24]. To initiate photo-polymerisation, the resist [24] (an 8:2 mixture of methacryloxypropyl trimethoxysilane, $99 \%$, Polysciences Inc. and zirconium $n$-propoxide, $70 \%$ in propanol, SigmaAldrich) contained the photo-initiator, (4, 4' - bisdiethylamino benzophenone, from SigmaAldrich, at a $1 \% \mathrm{w} / \mathrm{w}$ ) and following the polymerisation of the resist, it was developed in isopropyl alcohol for $\sim 10$ minutes to reveal the patterned structures. In order to produce a primary mould that had square holes, and hence a secondary PDMS mould that had upstanding square pillars, a DMD pattern that consisted of a square-ring/ frame (as shown in inset of Fig.4c) was used (100 pulses), and stitched together to form an overlapping 2D array of the structures. This is because unlike simple ablation wherein an imaged square would ablate and hence remove material to form a corresponding square hole, for the case of photopolymerisation (with a negative resist), a 'square' image pattern would result in the photopolymerisation of the corresponding square-area which then remains insoluble during subsequent development of the photo-polymer. This then would result in the formation of upstanding square pillars in the master, contrary to what is desired. Instead, if a 'square frame' is projected using the $\mathrm{DMD}$, since light is present only in the outer square-frame, (the white area in Fig.4(c) inset), it is only this exposed area that undergoes photo-polymerisation, and rendered insoluble during subsequent development. This will hence result in the formation of an array of wells in the master. Fig.4a shows a 2D array of wells created in the negative resist via DMD-photo-polymerisation using a square-frame pattern and Fig.4b shows the 2D array of the complementary pattern formed in the secondary PDMS mould using this master. Fig.4c shows the black/ white bitmap image of the square frame that was projected via the DMD onto the resist for photo-polymerisation. Fig.4c also shows the array of square frames that have been stitched together in the resist through a step-and-repeat illumination by the square frame pattern. The green square in the image is the fluorescence captured in 
real-time by the CCD camera used for the visualisation of the patterning procedure. As earlier, images shown in Fig.4a and Fig.4b have been acquired using an optical interference-based non-contact surface profiler.
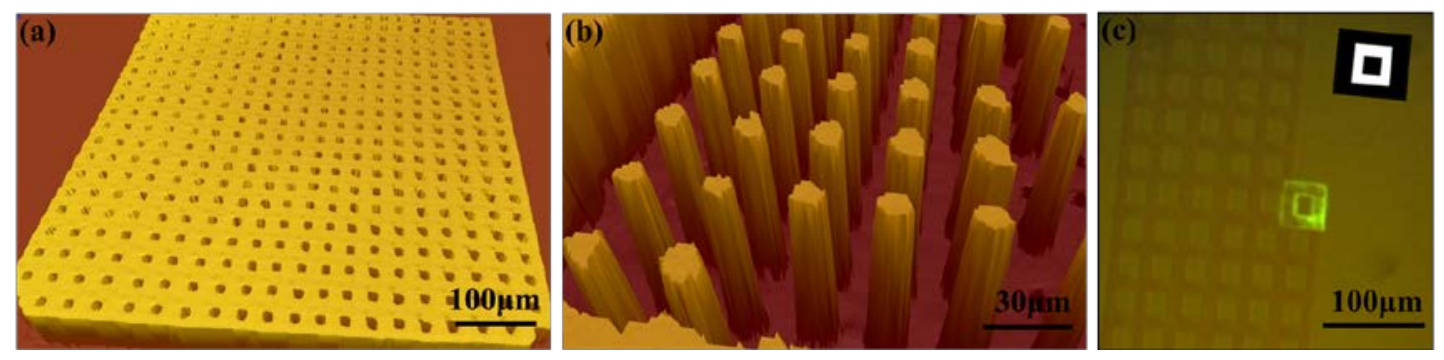

Figure 4: Images showing (a) a 2D array of wells formed via DMD-photo-polymerisation of a resist; (b) a 2D array of the complementary pattern formed in PDMS using the mould in (a), where both images have been taken using an optical interference-based non-contact surface profiler. Part (c) shows a CCD image of the step-and-repeat procedure in action, where the observed green fluorescence arises from illumination of a dye mixed in with the resist, for the purpose of alignment. The caption shows the DMD pattern that was used (white = laser light, black = no laser light). The heights of the upstanding PDMS structures in Fig. $4 \mathrm{~b}$ were measured to be $\sim 10 \mu \mathrm{m}$. As in Fig. $4 \mathrm{~b}$, they appear to be $>10 \mu \mathrm{m}$ because the images have been adapted through the profiler software for better visualisation.

\subsection{Micro-contact printing}

Finally, to demonstrate the usefulness of this PDMS structuring approach for micro-contact printing, this PDMS-mould, which was composed of an array of surface-relief pillars was used to contact-print a pattern onto a glass substrate. Fig.5a shows the secondary PDMS mould structured with an array of surface relief square patterns that were produced using the $\mathrm{Bi}_{2} \mathrm{Te}_{3}$ master mould formed via the DMD-based ablative route. The PDMS mask was first inked by dipping it for a few seconds in a black ink solution prepared by simple dilution in water. The inked PDMS mask was then contacted for a few tens of seconds with a glass slide by applying gentle pressure. The PDMS mask was detached and the ink pattern transferred onto the glass slide was imaged under an optical microscope. Fig.5b shows the optical microscope image of the array of square ink patterns produced to on the glass-slide.
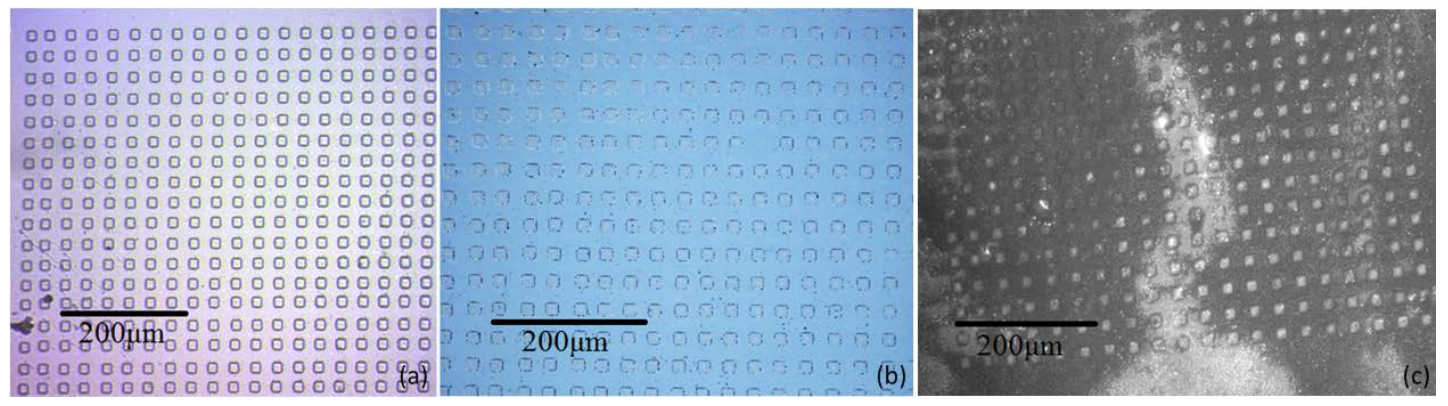

Figure 5: Images showing (a) PDMS-mask fabricated via DMD-ablation, (b) complementary ink-pattern and (c) fluorescent TRITC-labelled BSA contact printed on a glass-slide using this PDMS mould. 
Prior to the inking of the PDMS mould, to allow for adhesion of the water-based ink, it was necessary to render the PDMS surface hydrophilic, which required its treatment with oxygen (at 200 MTorr with a flow-rate of $90 \mathrm{sccm} / \mathrm{min}$ ) in a plasma etcher for $20 \mathrm{~s}$ at $20 \mathrm{~W}$. In a further experiment the same PDMS stamp was then used to contact-print an array of fluorescently-tagged biological molecules. Using a similar routine we were able to form an array of tetramethylrhodamineisothiocyanate (TRITC)-labelled Bovine Serum Albumin (BSA) on a pre-cleaned glass slide. $30 \mu \mathrm{l}$ of a TRITC-labelled BSA solution $(0.1 \mathrm{mg} / \mathrm{ml})$ was pipetted onto the PDMS stamp. The stamp was then kept in the dark for 30 minutes for the BSA to adsorb onto the PDMS stamp. Finally, the stamp was blow-dried with $\mathrm{N}_{2}$ and pressed onto a clean glass slide for $\sim 30 \mathrm{~s}$ to transfer the biomaterial. The contact-printed array was subsequently imaged using a fluorescent microscope. The tagged biomolecules were excited at a wavelength of $547 \mathrm{~nm}$ and the fluorescence at $572 \mathrm{~nm}$ was observed and imaged. Fig.5c shows an image captured using the fluorescence microscope. The white squares in Fig.5c are the TRICT-labelled BSA molecules that fluoresce and hence appear white in this black and white image captured via the fluorescence microscope. This shows that it was indeed possible to successfully contact-print the fluorescently tagged biomolecules without damaging them using the PDMS-stamp (shown in Fig.5a). Since the inked PDMS-stamp was pressed onto the glass slide using very slight finger-pressure, the pressure imparted through this manual procedure was non-uniform, which meant that some of the upstanding squares in the PDMSstamp were unable to contact the glass slide and hence transfer the biomolecules.

\subsection{Conclusions}

In conclusion, we have presented a rapid laser-based patterning procedure that employs a DMD in conjunction with a femtosecond laser for the patterning. The laser-based patterning route was used for the fabrication of the master mould either via a laser-based ablative procedure or a laser-based photo-polymerisation procedure. The laser-structured master was used to cast a PDMS mould that was then used to contact print an array of a fluorescently tagged biomolecule. The structures created via the ablative route had heights of $\sim 1 \mu \mathrm{m}$, and their minimum dimensions were $\sim 10 \mu \mathrm{m}$. For the photo-polymerisation based structuring route the dimensions of patterns produced were similar however, their heights were $\sim 10 \mu \mathrm{m}$. Using a step-and-repeat stitching procedure it was possible to structure patterns over areas with dimensions of $\sim 100 \mu \mathrm{m}$ by $\sim 300 \mu \mathrm{m}$. The DMD-based techniques presented here, are parallel structuring procedures and hence generally have a considerable speed advantage over serial point-by-point approaches. However, these approaches do not provide a route for 3D structuring. We believe this laser-based approach can be extended to form complex micronscale structures that are not only useful in contact printing applications but also in the fabrication of millimetre scale lab-on-chip fluidic devices. 


\section{Acknowledgements}

The authors are grateful to the Engineering and Physical Sciences Research Council (EPRSC) for funding via grants EP/J 008052/ 1 and EP/K023454/1.

\section{Figure captions}

Figure 1: Schematic of the DMD-based laser patterning set-up.

Figure 2: Schematic of the two DMD-based routes used to pattern a master via (a) ablation, and (b) multiphotonpolymerisation of the DMD-based laser patterning set-up.

Figure 3: Images (a) shows a 2D array of $10 \times 30$ shapes formed in a $\sim 1 \mu \mathrm{m}$ thick Bi2Te3 film via DMD-ablation. The black/ white inset shows the bitmap loaded onto the DMD for ablation of the Bi2Te3 film; (b) shows a complementary 2D array of 10×30 shapes (circles, triangles and squares) formed in PDMS using the Bi2Te3 mould in figure 3(a). Images have been taken using an interference-based surface profiler. The depths of the ablated features, and hence the corresponding heights of the upstanding PDMS structures was measured to be $\sim 1 \mu \mathrm{m}$. They however appear to be $>1 \mu \mathrm{m}$ because the images have been adapted through the profiler software for better visualisation.

Figure 4: Images showing (a) a 2D array of wells formed via DMD-photo-polymerisation of a resist; (b) a 2D array of the complementary pattern formed in PDMS using the mould in (a), where both images have been taken using an interference-based surface profiler. Part (c) shows a CCD image of the step-and-repeat procedure in action, where the observed green fluorescence arises from illumination of a dye mixed in with the resist, for the purpose of alignment. The caption shows the DMD pattern that was used (white = laser light, black = no laser light). The heights of the upstanding PDMS structures in Fig. 4b were measured to be $\sim 10 \mu \mathrm{m}$. As in Fig. 4b, they appear to be $>10 \mu \mathrm{m}$ because the images have been adapted through the profiler software for better visualisation.

Figure 5: Images showing (a) PDMS-mask fabricated via DMD-ablation, (b) complementary ink-pattern and (c) fluorescent TRITC-labelled BSA contact printed on a glass-slide using this PDMS mould. 


\section{References}

[1] J.C. McDonald, D.C. Duffy, J.R. Anderson, D.T. Chiu, H.K. Wu, O.J.A. Schueller, G.M. Whitesides, Fabrication of microfluidic systems in poly(dimethylsiloxane), Electrophoresis, 21 (2000) 27-40.

[2] S.K. Sia, G.M. Whitesides, Microfluidic devices fabricated in poly(dimethylsiloxane) for biological studies, Electrophoresis, 24 (2003) 3563-3576.

[3] Y.N. Xia, G.M. Whitesides, Soft lithography, Annu. Rev. Mater. Sci., 28 (1998) 153-184.

[4] A. Kumar, G.M. Whitesides, Features of gold having micrometer to centimeter dimensions can be formed through a combination of stamping with an elastomeric stamp and an alkanethiol ink followed by chemical etching, Appl. Phys. Lett., 63 (1993) 2002-2004.

[5] J. Garra, T. Long, J. Currie, T. Schneider, R. White, M. Paranjape, Dry etching of polydimethylsiloxane for microfluidic systems, J. Vac. Sci. Technol. A-Vac. Surf. Films, 20 (2002) 975-982.

[6] B.H. Jo, L.M. Van Lerberghe, K.M. Motsegood, D.J. Beebe, Three-dimensional microchannel fabrication in polydimethylsiloxane (PDMS) elastomer, J . Microelectromech. Syst., 9 (2000) 76-81.

[7] L. Miccio, M. Paturzo, A. Finizio, P. Ferraro, Light induced patterning of poly(dimethylsiloxane) microstructures, Opt. Express, 18 (2010) 10947-10955.

[8] W.R. Childs, R.G. Nuzzo, Decal transfer microlithography: A new soft-lithographic patterning method, J. Am. Chem. Soc., 124 (2002) 13583-13596.

[9] A.L. Thangawng, M.A. Swartz, M.R. Glucksberg, R.S. Ruoff, Bond-detach lithography: A method for micro/ nanolithography by precision PDMS patterning, Small, 3 (2007) 132-138.

[10] M.S. Thomas, B. Millare, J.M. Clift, D. Bao, C. Hong, V.I. Vullev, Print-and-Peel Fabrication for Microfluidics: What's in it for Biomedical Applications?, Ann. Biomed. Eng., 38 (2010) 21-32.

[11] J.C. McDonald, M.L. Chabinyc, S.J. Metallo, J.R. Anderson, A.D. Stroock, G.M. Whitesides, Prototyping of microfluidic devices in poly(dimethylsiloxane) using solid-object printing, Anal. Chem., 74 (2002) 1537-1545.

[12] J.C. McDonald, G.M. Whitesides, Poly(dimethylsiloxane) as a material for fabricating microfluidic devices, Accounts Chem. Res., 35 (2002) 491-499.

[13] Y.N. Xia, G.M. Whitesides, Soft lithography, Angew. Chem.-Int. Edit., 37 (1998) 551-575.

[14] H. Selvaraj, B. Tan, K. Venkatakrishnan, Maskless direct micro-structuring of PDMS by femtosecond laser localized rapid curing, J . Micromech. Microeng., 21(2011).

[15] S. Rekstyte, M. Malinauskas, S. J uodkazis, Three-dimensional laser micro-sculpturing of silicone: towards bio-compatible scaffolds, Opt. Express, 21(2013) 17028-17041.

[16] R. Buividas, S. Rekstyte, M. Malinauskas, S. Juodkazis, Nano-groove and 3D fabrication by controlled avalanche using femtosecond laser pulses, Opt. Mater. Express, 3 (2013) 16741686.

[17] R.R. Gattass, E. Mazur, Femtosecond laser micromachining in transparent materials, Nat. Photonics, 2 (2008) 219-225.

[18] M.D. Shirk, P.A. Molian, A review of ultrashort pulsed laser ablation of materials, J . Laser Appl., 10 (1998) 18-28.

[19] S. Maruo, J.T. Fourkas, Recent progress in multiphoton microfabrication, Laser Photon. Rev., 2 (2008) 100-111.

[20] S. Maruo, O. Nakamura, S. Kawata, Three-dimensional microfabrication with twophoton-absorbed photopolymerization, Opt. Lett., 22 (1997) 132-134.

[21] B. Mills, M. Feinaeugle, C.L. Sones, N. Rizvi, R.W. Eason, Sub-micron-scale femtosecond laser ablation using a digital micromirror device, J . Micromech. Microeng., 23 (2013) 035005. [22] T. Instruments, in.

[23] Zemetrics, in.

[24] A. Ovsianikov, J. Viertl, B. Chichkov, M. Oubaha, B. MacCraith, I. Sakellari, A. Giakoumaki, D. Gray, M. Vamvakaki, M. Farsari, C. Fotakis, Ultra-Low Shrinkage Hybrid Photosensitive Material for Two-Photon Polymerization Microfabrication, ACS Nano, 2 (2008) 2257-2262. 